\title{
Managing acute disturbance in the context of COVID-19
}

\section{0}

Revised 15 December 2020 [major revision: 5.23, 5.40, 5.41, 5.49, 5.55, 5.62-5.67, 6.1-6.10 new; $1.9,2.3,2.5,2.13-2.15,3.3,3.10,4.1,4.19,5.18,5.22-5.81$, Appendix 2 updated] Revised 11 May [2.5 updated]

Revised 10 April [2.21-2.23, 5.53-5.71 added; 5.52 updated]

Revised 31 March 2020 [3.12 added]

Revised 26 March 2020 [4.7, 4.19 updated] 


\section{Contents}

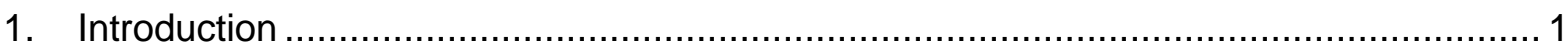

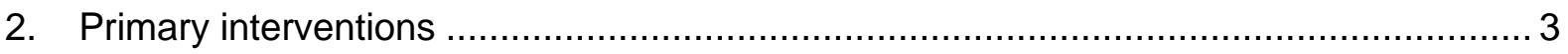

Local ethics committees ....................................................................................... 4

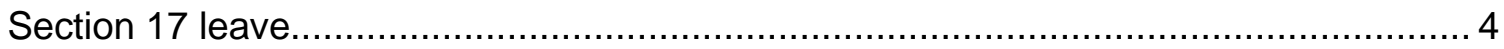

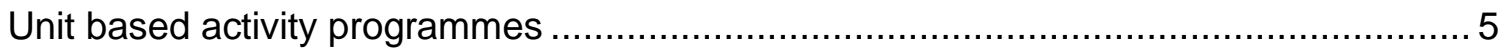

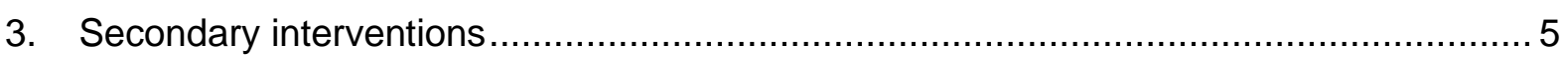

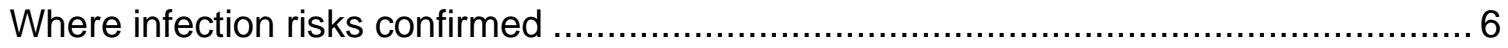

Isolation and COVID-19 infection risk secondary intervention ................................. 6

4. Legal and ethical considerations in respect of secondary and tertiary intervention ......... 7

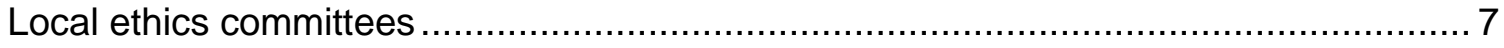

Mental Health Act Code of Practice (MHA CoP 2015) ........................................... 7

Potential areas where risk COVID-19 infection could result in a cogent reason to depart

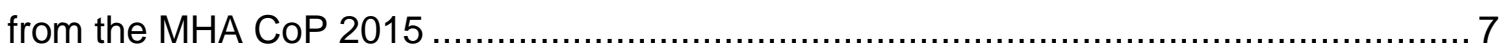

Mental Capacity Act (MCA)/DoLS ................................................................ 9

5. Tertiary intervention infection risk, acute disturbance and active resistance ................. 9

Location and review of segregation/seclusion .................................................... 10

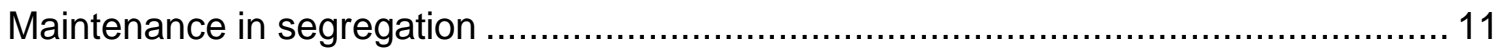

Medication use for acute disturbance ........................................................... 12

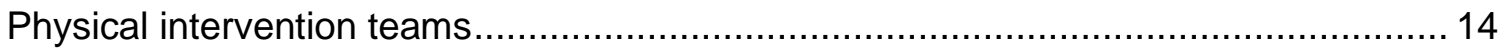

Personal protective equipment (PPE) and physical intervention (PI) ........................ 15

Preliminary tests of protective equipment shown to be viable in circumstances where resistance to direction and/or physical intervention is required ................................. 15

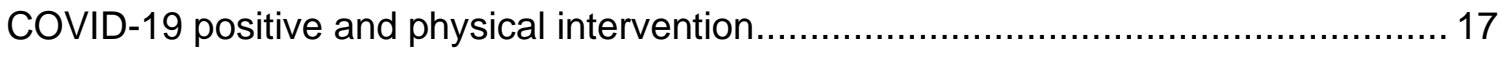

PPE shown as viable in physical intervention test scenarios ................................. 18

Recommended physical intervention PPE set ....................................................... 18

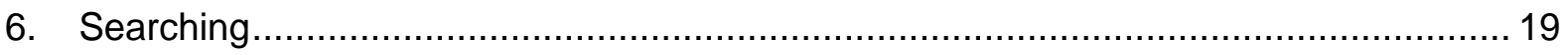

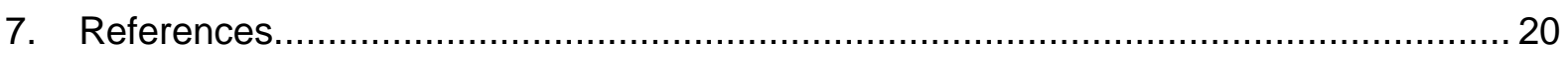




\section{NAPICU Guidance}

\section{Managing acute disturbance in the context of COVID-19}

Editor Roland Dix, Consultant Nurse and Approved Clinician

Associate Editor Tom Tunnicliffe, Consultant Nurse and Approved Clinician

Technical Editor Dr Sally Thomas, Journal of Psychiatric Intensive Care

\section{INTRODUCTION}

1.1. The current national situation arising from COVID-19 will present challenges for the care and engagement of mental health patients presenting with acute disturbance and who are also a possible infection risk.

1.2. Along with our colleagues in general medicine, mental health staff will be determined to provide high quality care to our patients and demonstrate our ability to contribute to the wider national public service effort underway during this challenging period.

1.3. As with current practice, it is important that restrictive interventions are kept to the minimum necessary. Also, that managing acute disturbance in the context of infection risk is underpinned by the usual levels of proportionality, balance and compassion, and does not create difficulties that could otherwise have been avoided.

1.4. It is important to acknowledge than many of us working in inpatient mental health will already have had experience of supporting patients who may be challenging, and at the same time have medical concerns including infection risk.

\section{Initial frame work of considerations}

1.5. As experience increases, the approaches and techniques that are effective for supporting patients experiencing acute disturbance who also present infection risks will improve.

1.6. Of particular concern, are those who are experiencing acute disturbance and who also:

- Represent COVID-19 infection risk to others or

- Are in a high risk group for infection or

- Are in a ward that is 'locked down' or 'self isolating'. 


\section{Legal and ethical considerations}

1.7. Consistent with national guidance, each provider should set up local ethics committees that are able to consider any restrictive interventions employed for managing COVID-19 infection risks including restriction of leave.

1.8. The interventions and management plans that may be required to safely care for this particular group of patients whilst also minimising the risk of spreading the infection could present ethical and legal challenges with respect to professional practice, the Mental Capacity Act and the Mental Health Act.

1.9. The following guidance represents a description of the issues that may be helpful. Each Provider Organisation will be aware of national policy developments and will have developed local procedures and policies which are not superseded by this guidance. This guidance aims to provide a guide to considering some of the ethical, legal and practice issues.

1.10. The following represents an overall framework which is intended to be of assistance in concert with nationally and locally agreed practice.

1.11. It is expected that as experience of supporting patients exhibiting acute disturbance who may also represent a COVID-19 infection risk increases, national, local and professional guidance will be further revised, amended and developed.

\section{NAPICU COVID-19 online platform}

1.12. A page is available on the NAPICU website to provide a platform for providers of PICU and other related services to share their experience and disseminate experiencebased learning amongst the PICU clinical community.

1.13. Use the platform to share any experience you have that may be helpful to others with:

- Supporting a patient presenting acute disturbance and COVID-19 infection risk

- Isolation or segregation of those patients who have issues associated with COVID-19 infection

- Managing mental health inpatient scenarios in the context of COVID-19.

1.14. Write a summary of up to 300 words and e-mail it to info@napicu.org.uk.

1.15. Summaries will be posted on the NAPICU COVID-19 practice page. 


\section{Hierarchy of response for acutely disturbed patients who may also be an infection risk regarding COVID-19}

1.16. Interventions for supporting an acutely disturbed patient should be divided into:

- Primary

- Secondary and

- Tertiary interventions.

1.17. This is a similar approach to engaging with patients who do not present an infection risk of COVID-19, although the additional considerations arising from infection risk are the focus of this guidance.

\section{PRIMARY INTERVENTIONS}

\section{Information}

2.1. Many patients and staff may be fearful of the COVID-19 situation; such anxieties can be also be very infectious. Caution should be exercised so as not to exacerbate an already difficult situation.

2.2. One paper from the experience of a mental health inpatient service in Wuhan China made several recommendations on COVID-19 issues to consider. Please review Zhu et al. (2020) ${ }^{1}$ (full reference provided below and linked here).

2.3. Experience suggests that many mental health inpatients may often be relatively detached from what is happening in the wider community. As the pandemic has progressed, a wide variety of views have developed within society with some people assigning more importance to the risk of COVID infection than others.

2.4. This may require effort from staff, to convey the seriousness of the situation requiring action, while at the same time not raising fear or frustration to the extent that create further problems with cooperation and engagement.

2.5. Following guidance issued by NHS England (27 April 2020) all admissions to hospital should be screened for COVID-19. This includes completing a swab and isolating symptomatic patients. The requirement to isolate relates only to symptomatic patients and those confirmed COVID-19 positive; asymptomatic patients who are awaiting the

\footnotetext{
${ }^{1}$ Zhu, Y., Chen, L., Ji, H., Xi, M., Fang, Y. and Li, Yi (2020) The risk and prevention of novel coronavirus pneumonia infections among inpatients in psychiatric hospitals. Neuroscience Bulletin, 36: 299-302. https://doi.org/10.1007/s12264-020-00476-9
} 
results of a swab will be advised to self-isolate and follow social distancing guidance until results are obtained. The legal frameworks which support isolation are presented below. Swabbing advice now recommends swabbing on admission (day 1), day 3 and day 5 (NHS England 2020a) ${ }^{2}$.

2.6. Patients admitted to the PICU should be engaged in a process of discussion and information sharing about COVID-19 infection risk. The possibility should be addressed that a patient who has identified infection risk issues, may need to be subject to isolation, possibly within a PICU environment.

2.7. A formal capacity assessment regarding this discussion should be completed and recorded in the patient record.

2.8. The locally derived agreed location for engaging with a patient presenting infection risk should be identified and discussed with the patient. A brief description of the associated isolation procedures should be offered with the intention of, so as far as is possible, achieving cooperation or minimal resistance should these procedures be required. This is a procedure similar to developing an 'advance statement'.

2.9. This could include an information leaflet which outlines the main issues including the potential need for personal protective equipment (PPE) to be implemented.

2.10. This may also include other infection control measures e.g. provision of personally allocated utensils for dietary and fluid intake consistent with national and local infection control protocols.

\section{Local ethics committees}

2.11. Consistent with national guidance, each provider should set up local ethics committees that are able to consider any restrictive interventions employed for managing COVID-19 infection risks including restriction of leave.

\section{Section 17 leave}

2.12. Leave outside needs to balance risks and benefits in-line with government advice and take into account the innate issues of operating an inpatient mental health unit.

\footnotetext{
${ }^{2}$ NHS England (2020a) Key actions: infection prevention and control and testing. 17 November. https://www.england.nhs.uk/coronavirus/publication/key-actions-infection-prevention-and-control-andtesting/
} 
2.13. Time spent outside, including Section 17 leave, should be consistent with national guidance which is issued by the government and can change relatively quickly.

2.14. Expected procedures for returning from escorted and unescorted leave, for example search and hygiene procedures, should be explained and implemented. For further consideration of searching see $6.1-6.10$ below.

2.15. While engaged in Section 17 leave (escorted or unescorted), social distancing, use of masks, locations that are recommended for visiting, and those that are not recommended (avoiding crowded areas) must be clearly be identified.

2.16. Some services may be on the site of District General Hospitals with coffee shop and other canteen facilities that may ordinarily provide a visiting location for mental health inpatients. For the period of the epidemic, these locations should be avoided.

2.17. Where there is doubt, reference should be made to the local ethics committee.

\section{Unit based activity programmes}

2.18. Unit based activity programmes are useful for minimising disturbance and improving cooperation, which will contribute to infection control management.

2.19. Infection control measures should be consistent with national and local guidance.

2.20. As access to facilities areas off the unit diminish, resources to provide unit-based activity should be given equal status to other priorities.

2.21. Condense use of materials, objects and tools to those that can be wiped clean and disinfected, and those which can be disposed after one use.

2.22. Small group-based interventions should be provided in areas large enough to adhere to social distancing requirements, e.g. outside or in large enough rooms that are regularly cleaned.

2.23. For patients in self-isolation, provide packs of activities that can be done in their bedrooms, ensuring activities are achievable for each individual's level of ability.

\section{SECONDARY INTERVENTIONS}

3.1. The unit should have a clear method of identification of patients who may present risk if infected either to themselves or to others. This should be based on a robust checklist of symptoms and COVID-19 testing wherever this is possible.

3.2. A systematic approach is required to avoid patients being unnecessarily subject to placement or procedures that result in a finite resource being inefficiently deployed. 
3.3. Suggested methods could include daily monitoring of temperatures and enquiry/observation to ascertain the presence of a cough, or loss of taste or smell. Practice in this area is rapidly developing. Testing should occur wherever the criteria is met for doing so.

3.4. The identification of 'high risk' or vulnerable patients as described by Public Health England is recommended to allow for a graded approach to monitoring physical health and directing management plans.

\section{Where infection risks confirmed}

3.5. From the point at which infection risks have been confirmed (risk to others or high-risk group if infected) a specific care plan of intervention and engagement, taking into account the specific mental and behavioural pathology associated with the patient should be devised.

3.6. This should include a hierarchy of response as illustrated in Appendix 1.

3.7. For those experiencing mental and behavioural disturbance although who are for the time being, generally able to follow direction and cooperate, should be maintained in an area or zone consistent with local procedures for isolation of these presenting infection risk. For PICU patients, this may be designated areas within the unit.

\section{Isolation and COVID-19 infection risk secondary intervention}

3.8. For those subject to isolation, an assessment should be made of items available to the patient which could improve cooperation and experience of isolation, reducing the potential for disturbance.

3.9. This may require re-assessment of the items of concern/restricted items list generally operated by the unit.

3.10. Items helpful in meaningfully occupying time should be allocated for the patient's individual use, and not re-introduced to general unit use until cleaning, quarantine of items or disposal consistent with infection control recommendations.

3.11. Any items that can be disposed of following use should be disposed of within infection control advice.

3.12. The care plan supporting isolation should have provision for recognising and dealing with any physical deterioration related to the known course of COVID-19, or for other reasons. Local policies on the management of physical health in confirmed/suspected COVID-19 cases should be followed. 


\section{LEGAL AND ETHICAL CONSIDERATIONS IN RESPECT OF SECONDARY AND TERTIARY INTERVENTION}

4.1. This guidance does not provide authority for patients presenting risk of infection to others with COVID-19 to be forcibly segregated or secluded for this risk alone. Least restrictive options must be employed wherever possible and risk of infection cannot be completely eradicated.

4.2. Just as is the case with our general medical colleagues, some degree of risk is unavoidable. The nationally recommended ethics committees will be required to carefully balance risk with the use of restrictive interventions.

\section{Local ethics committees}

4.3. Consistent with national guidance, each provider should set up local ethics committees that are able to consider any restrictive interventions employed for managing COVID-19 infection risks. If in doubt regarding any isolation, segregation or seclusion issue, refer to the local ethics committee.

\section{Mental Health Act Code of Practice (MHA CoP 2015)}

4.4. Chapter 26 of the Mental Health Act Code of Practice (MHA CoP 2015; Department of Health 2015$)^{3}$ governs the use of restrictive interventions.

4.5. Wherever possible, adherence to the Code should be maintained. Only where there is a cogent reason should there be a departure from the Code.

\section{Potential areas where risk COVID-19 infection could result in a cogent reason to depart from the MHA CoP 2015}

4.6. Where a cogent reason can be documented to depart from the MHA CoP this can be acceptable. This should be supported by the local ethics committee.

4.7. The application of the MHA CoP 2015 should be considered in the context of The Coronavirus Act 2020 (in particular Schedule 21) detailing 'Powers relating to potentially infectious persons'.

\footnotetext{
${ }^{3}$ Department of Health (2015) Mental Health Act 1983: Code of practice. 
4.8. This legislation includes powers that provide the Secretary of State and Public Health Consultants to authorise the testing and isolation of persons with suspected or confirmed COVID-19.

4.9. Whilst such measures may not be considered commensurate to those with the MHA CoP 2015, they may offer a cogent reason to depart and provide rationale for this.

4.10. Where such departures from the MHA CoP 2015 are required, every effort should be made to ensure the principles of the MHA CoP 2015 are followed. This includes that when restrictive measures are required they should be planned, evidence based, lawful, in the patient's interests, proportionate and dignified (Department of Health 2015).

4.11. Once it has been appropriately established that the patient represents a significant risk of infection, this can provide the basis for extended isolation for the period of the infection risk.

4.12. For a patient representing risk of infection, an individual care plan should be developed with the aim of maintaining cooperation with isolation and diminishing the need for physical intervention (PI) or other restrictive practices (as detailed above). All effort should be made to achieve agreement and cooperation.

4.13. Where isolation is required solely for infection control reasons this may not represent the need for review and monitoring of seclusion as described in Chapter 26 of the MHA CoP 2015 (Department of Health 2015; CQC 20204).

4.14. If the patient verbally disagrees with the care plan, although are not actively resisting it, then this may not represent the need for review and monitoring of seclusion as described in Chapter 26 MHA CoP 2015.

4.15. Where risk of infection has been robustly established, it should be considered as a cogent reason to depart from the Code's definition of seclusion providing that the patient is willing to cooperate and/or not physically actively resist the care plan of isolation.

4.16. If in doubt regarding any isolation, segregation or seclusion issue, refer to the local ethics committee.

\footnotetext{
${ }^{4}$ CQC (2020) Monitoring the Mental Health Act in 2019/20: The Mental Health Act in the COVID-19 pandemic. Care Quality Commission.

https://www.cqc.org.uk/sites/default/files/20201127_mhareport1920_report.pdf
} 


\section{Mental Capacity Act (MCA)/DoLS}

4.17. The Mental Capacity Act (MCA) is used when an individual lacks the mental capacity to make a specific decision. In regard to COVID-19, this could include decisions relating to their care e.g. the patient does not understand the need to use oxygen therapy to help their breathing. Staff can make a best interest decision on behalf of their patient unless there is a Health and Welfare Attorney or Court Appointed Deputy who can be contacted to make the decision.

4.18. In an emergency situation, treat first unless there is awareness of a legitimate advance decision to the contrary. Proportionate restriction or restraint, which that does not amount to a 'deprivation of liberty', is permitted under the Mental Capacity Act for the protection of the individual.

4.19. The MCA is not used for the protection of others; e.g. if the decision relates to the patient's understanding of the need to remain quarantined for the protection of others, Public Health Law (such as the The Coronavirus Act 2020) would apply and a record should be made in the patient's clinical records that the patient's liberty is restricted for the wider public interest.

4.20. Patients suspected of infection with COVID-19 who themselves represent an infection risk should be isolated for a period of 7 days.

\section{TERTIARY INTERVENTION INFECTION RISK, ACUTE DISTURBANCE AND ACTIVE RESISTANCE}

\section{Assessment}

5.1. In rare circumstances, it is possible that a person who is positive for COVID-19 and experiencing acute mental and behavioural disturbance, or for other reasons e.g. personality disorder, may recklessly, or in extreme cases deliberately, increase infection risk to others.

5.2. In such circumstances it is possible that this may involve actively and persistently physically resisting the isolation care plan.

5.3. In these circumstances, such actions could be considered as disturbed behaviour in the context of their mental condition representing a significant risk to others. 
5.4. This should be considered along with the other risk behaviours that may in themselves also provide the basis for seclusion/segregation and fall under the safeguards detailed in the MHA CoP 2015.

\section{Location and review of segregation/seclusion}

5.5. For the period of time that the person with COVID-19 presents with behaviour that is a significant risk to others, which could include an infection risk, and lesser restrictive intervention is not possible, then consideration should be given to extended segregation.

5.6. While all effort should be made to avoid the need for tertiary interventions, the balance is pushed toward the use of segregation and/or seclusion, where close physical contact such as extended holds are the only alternative.

\section{Location of segregation}

5.7. Methods of segregation may vary between Provider Organisations depending on the format of Extra Care Areas, seclusion rooms and other facilities for engaging with acute disturbance.

5.8. This is largely consistent with existing application of the MHA CoP 2015 with infection risk considered additional risk behaviour. This would also be consistent with those who present behavioural disturbance and risk of infection other than COVID-19, for example, hepatitis.

5.9. It is possible that there may be no alternative to using bedrooms or locking off areas of a unit or ward.

5.10. For facilities that do not have provision for hatch feeding or other similar methods, then personal protective equipment (PPE) should be used at every point of potential infection.

\section{Local ethics committees}

5.11. Consistent with national guidance, each provider should set up local ethics committees that are able to consider any restrictive interventions employed for managing COVID-19 infection risks. If in doubt regarding any isolation, segregation or seclusion issue, refer to local ethics committee. 


\section{Maintenance in segregation}

5.12. Specific care plans around diet, fluid intake and activities of daily living should be developed with a particular focus on diminishing opportunities for infection and transmission.

5.13. Segregation reviews should take account of risk of infection and avoid close proximity with the staff wherever possible. It is possible, that due to staff shortages, the specific grade and profession of staff recommended by the MHA CoP 2015 to undertake reviews will not be available.

5.14. In this case, the review intervals and considerations should take place with staff that are available making all effort to maintain the safeguards of the Code.

5.15. As this scenario may represent a departure from the MHA CoP 2015, clear and robust multidisciplinary documentation should be completed to include a rationale, justification and management plan.

5.16. It is of the utmost importance that an ethical balance is maintained safeguarding the patient and others. This will require careful thought in difficult circumstances on a case by case basis. Where infection control is a major concern for any segregated patient, account should be taken of the infection period duration during the reviews.

5.17. The care plan supporting isolation or segregation should have provision for recognising and dealing with any physical deterioration related to the known course of COVID-19, or for other reasons.

\section{Transition from segregation to isolation}

5.18. All effort should be made to achieve cooperation and removal of the need for enforced segregation.

5.19. This should be considered at the regular reviews required by the MHA CoP 2015.

5.20. Disturbed, uncooperative or aggressive behaviour that may also represent an infection risk, should form part of the process of review for the need for extended segregation/seclusion.

5.21. As soon as possible, segregation should be discontinued in favour of lesser restrictive isolation where infection risk remains. 


\section{Medication use for acute disturbance}

5.22. The choice of medication should follow your own Trust, NICE or Joint BAP NAPICU guidance (Patel, Sethi et al. 2018) ${ }^{5}$ but require some additional consideration of the specific contra-indications and side effects (see below) that are known to occur with COVID-19 and other infections. Importantly, the current physical health of the patient is a key factor in the choice.

5.23. Due to the nature of COVID-19, patients may be at a higher risk of delirium. If delirium is suspected, then delirium guidance should be followed (BGS 2020).

5.24. If a patient with suspected or diagnosed COVID-19 is acutely disturbed, and there are no signs of respiratory compromise (decreased or increased respiratory rate), cardiovascular disease and/or decreased level of consciousness; then medication should be used with caution as the full effects of COVID-19 are still not fully understood.

5.25. Choose short-acting medication as a patient's physical health condition may rapidly deteriorate. Ensure the medication for acute disturbance is an effective dose as an ineffective dose may lead to the increased need for additional injections.

5.26. Where possible, oral medication is preferred and should be offered as the first choice. Parenteral medication is more likely to cause dose-related side effects such as respiratory depression, sedation, postural drop, QTc prolongation and extra-pyramidal side effects (EPS).

5.27. COVID-19 can affect the respiratory function of patients. Psychotropic medications, especially benzodiazepines, can cause respiratory depression. Benzodiazepines should not be used when a patient has acute pulmonary insufficiency.

5.28. Lorazepam would be the preferred benzodiazepine due to its shorter half-life. Simultaneous injections of olanzapine and benzodiazepines can result in excessive sedation and cardiorespiratory depression so must be given at least an hour apart. Ensure immediate access to flumazenil is available if benzodiazepines are given.

5.29. If there is evidence of cardiovascular disease, including a prolonged QTc interval, or no recent electrocardiogram (ECG), avoid intramuscular haloperidol combined with

\footnotetext{
${ }^{5}$ Patel, M.X., Sethi, F. et al. (2018) Joint BAP NAPICU evidence-based consensus guidelines for the clinical management of acute disturbance: de-escalation and rapid tranquillisation. Journal of Psychiatric Intensive Care, 14: 89-132. https://doi.org/10.20299/jpi.2018.008

${ }^{6}$ BGS (2020) Coronavirus: Managing delirium in confirmed and suspected cases. British Geriatrics Society. Updated 25 March. https://www.bgs.org.uk/resources/coronavirus-managing-delirium-inconfirmed-and-suspected-cases
} 
intramuscular promethazine. Consider intramuscular lorazepam or intramuscular olanzapine.

5.30. Febrile individuals with a history of seizures may have their seizure threshold altered by some medications. Medical advice should be sought if there is any doubt.

5.31. All antipsychotics can cause neuroleptic malignant syndrome (NMS). If NMS occurs, immediately discontinue antipsychotics and other drugs that may contribute to the underlying disorder, monitor and treat symptoms, and treat any concomitant serious medical problems.

5.32. Inhaled loxapine is contra-indicated in patients with acute respiratory distress or with active airways disease and with the current use of medications to treat airways disease. Therefore, inhaled loxapine should be avoided in suspected or confirmed cases of COVID-19.

5.33. Physical health monitoring, especially respiratory rate and level of consciousness, should be carried out when either oral or parenteral rapid tranquillisation (RT) is given. Oxygen saturation levels are recommended for patients who receive RT but are especially important in COVID-19 as they have been shown to be an early detection method for deterioration of COVID-19 (NHS England 2020b) ${ }^{7}$.

\section{Other COVID-19 medication issues}

5.34. A COVID-19 vaccine has recently been licenced and other vaccines are in the process of being licenced. At present there are no data sheets or company information listing the contra-indications, warnings, interactions or side effects.

5.35. Many of the other treatments for COVID-19 focus on alleviating associated symptoms. The position is being further developed as the effects of COVID-19 become better understood.

5.36. There are many different types of treatments in research: lopinavir/ritonavir, remdesivir, favipiravir, chloroquine, hydroxychloroquine, nitazoxanide, ribavirin and dexamethasone. There is growing evidence for some of the treatments but no licensed preparation has emerged. Many of these agents have drug interactions and advice regarding these should be sought from the pharmacy team or from http://www.covid19druginteractions.org.

\footnotetext{
${ }^{7} \mathrm{NHS}$ England (2020b) Pulse oximetry to detect early deterioration of patients with COVID-19 in primary and community care settings. 11 June. https://www.england.nhs.uk/coronavirus/wpcontent/uploads/sites/52/2020/06/C0445-remote-monitoring-in-primary-care-revised.pdf
} 
5.37. There is currently no strong evidence that ibuprofen can make COVID-19 worse. When starting treatment for fever or pain in COVID-19, consider all available treatment options, including paracetamol and NSAIDs. Paracetamol should be used as a first treatment option for fever or pain, unless contra-indicated. If a patient is already taking ibuprofen or another non-steroidal anti-inflammatory (NSAID) review the prescription $\left(\right.$ NICE 2020) ${ }^{8}$.

5.38. Other treatments are based on treatment of secondary infections or symptoms and may include antibiotics, venous thromboembolism (VTE) therapy, and nebulisers (e.g. salbutamol and/or ipratropium) and/or oxygen.

5.39. Be aware of drug interactions in patients prescribed physical health treatments; e.g. clarithromycin can prolong the QTC and should be used with caution with antipsychotics.

5.40. There is no evidence to support taking vitamin D supplements to specifically prevent or treat COVID-19. However, all people should continue to follow UK Government advice on daily vitamin $\mathrm{D}$ supplementation to maintain bone and muscle health during the COVID-19 pandemic (NICE 2020b) ${ }^{9}$.

\section{Physical intervention teams}

5.41. The following guidance has been informed by a systematic evaluation of the performance of PPE and procedures used for physical intervention (PI). This included contact contamination and other characteristics of PPE. Further details of this are included in Appendix 2. The following should be considered in concert with, but not supersede, any local arrangements.

5.42. Consideration should be given to identifying a PI team who may become more familiar with the use of PPE for those at risk of infection.

5.43. Consideration should be given to diminishing the psychiatric emergency response in terms of the number of PI practitioners, and wherever possible this should be focused on the minimum number of people required to manage the situation. This may assist in reducing the spread of infection.

\footnotetext{
${ }^{8}$ NICE (2020a) Acute use of non-steroidal anti-inflammatory drugs (NSAIDs) for people with or at risk of COVID-19. Evidence Summary ES23. 14 April. https://www.nice.org.uk/advice/es23/chapter/Factors-for-decision-making 9 NICE (2020b) Vitamin D for COVID-19. Evidence Summary ES28. 29 June. https://www.nice.org.uk/advice/es28/chapter/Advisory-statement-on-likely-place-in-therapy
} 
5.44. In due course, it may be possible to identify staff who have developed some immunity to COVID-19 as a result of vaccination.

\section{Personal protective equipment (PPE) and physical intervention (PI)}

5.45. There may be circumstances that, for the time being, acute disturbance representing risks could result in the need for PI to maintain the protection for others.

5.46. In addition to PPE, post-PI cleansing procedures (see 5.60-5.67 below) are important for engaging with a patient who may be behaviourally disturbed, resistive or who requires $\mathrm{PI}$.

5.47. The availability of PPE is a priority for health care providers.

5.48. Infection control advice should be followed with regard to managing PPE following PI with a high-risk patient. Amongst other procedures this may involve leaving work in different clothes to those worn in higher risk activities.

5.49. It is important that items of PPE are properly fitted to the individual. This includes appropriate sizing options as well as adjustment of straps etc.

\section{Preliminary tests of protective equipment shown to be viable in circumstances where resistance to direction and/or physical intervention is required}

\section{Face masks (FPP3 and FRSM)}

5.50. Face masks have proved viable in PI scenarios without presenting significant difficulties other than a tendency for the wearer to experience mild discomfort due to raised temperature and/or a sensation of restriction resulting from the mask.

\section{Eye/spit protection}

5.51. Preliminary tests have indicated that appropriately fitted goggles are effective when used in the $\mathrm{PI}$ activity. Risk of condensation resulting in diminished vision requires mitigation. This can be achieved by the design of the eye protection or preparation with anti-misting sprays.

5.52. There can be issues with visor-type face protection being dislodged during episodes of $\mathrm{PI}$ which may require the availability of another person to replace headwear for those engaged in implementing holds. 


\section{Aprons}

5.53. Preliminary tests indicate that aprons provide hindrance to those engaged in PI and become easily displaced, ripped off and thereafter providing a slip hazard. At this stage, significant caution should be given to the use of aprons.

\section{Scrubs}

5.54. Scrubs have shown to be effective during tests, in providing some protection while not representing obstacles to $\mathrm{PI}$. Scrubs are recommended for PI teams. Wherever possible (in the case of planned $\mathrm{Pl}$ ) a second layer of scrubs which can be removed following $\mathrm{PI}$ is recommended. Disposable scrubs have the advantage of easier doffing.

5.55. Where a second layer is not possible (in the case of emergency, unplanned PI) clothing should be changed following PI.

\section{Gloves}

5.56. Nitrile gloves have proved effective during simulated PI testing.

5.57. Gloves could represent an increased risk of pinching the skin for those subject to holds resulting from the increased grip that can be achieved from the glove over that that would normally be experienced by the naked hand.

5.58. This should be a consideration within PI procedures.

\section{Disposable overalls}

5.59. Coveralls provide high levels of protection although also have disadvantages which should be considered. These include:

- Overheating of the staff member especially during a prolonged episode of PI

- Greater difficulty in donning and doffing

- Visual impact on an already distressed, hostile and/or frightened patient.

\section{Cleaning and disposal of equipment following physical intervention with a person representing an infection risk}

5.60. Reusable equipment should be cleaned or quarantined consistent with infection control procedures after every episode of PI.

\section{Personal cleansing post-PI}

5.61. Following doffing, it is important that staff members engage in thorough personal cleansing. This should take place as soon after PI as practicable. 
5.62. Where possible, this should be undertaken with soap and water in appropriate washing facilities. In many locations where PI has taken place, this may not be possible without travelling through other areas or having to open doors etc. Where this is the case, disposable body wash wipes should be used.

\section{Cleansing with disposable wipes.}

5.63. The procedure for cleansing with wipes should be described on an action card agreed with local infection control specialists.

5.64. Individual wipes should be used for each arm starting with the elbow and working down to the wrist. Following this another wipe should be used for the hands.

\section{Cleansing face and neck}

5.65. Wipes designed and specified for face and neck should be used. These may be different from those specified for hands and arms.

5.66. At the conclusion of arms and hands cleansing, the face should be cleansed starting at the top working down over the eye lids with eyes closed to below the chin.

5.67. A separate wipe should be used for the neck, working from the front of the neck backwards.

\section{COVID-19 positive and physical intervention}

5.68. National Guidance on the use of PPE in different care settings in a COVID-19 context is detailed on the Public Health England website ${ }^{10}$.

5.69. Undertaking PI with a person confirmed as COVID-19 positive generates specific risks associated with close proximity/physical contact, potential for shouting/spitting/biting and increased potential for transmission of oral fluids.

5.70. Undertaking PI with a person who is suspected or confirmed as COVID-19 positive should be considered one of the highest infection risk procedures that will be carried out in the acute mental health in-patient context.

5.71. There has been debate regarding the extent to which COVID-19 is airborne (Morawska \& Milton 2020) ${ }^{11}$. Appendix 2 details characteristics of PI which may increase risk of COVID-19 infection by non-contact means.

\footnotetext{
10 Public Health England (2020) updated 31 July. https://www.gov.uk/government/collections/coronavirus-covid-19-personal-protective-equipment-ppe 11 Morawska, L. and Milton, D.K. (2020) It is time to address airborne transmission of coronavirus disease 2019 (COVID-19). Clinical Infectious Diseases, 71(9): 2311-2313. https://doi.org/10.1093/cid/ciaa939
} 
5.72. Whilst such debates continue, NAPICU recommends that an approach which offers staff the greatest feasible protection is followed.

5.73. There are times when $\mathrm{PI}$ is planned. During these times, it is recommended that a specific PI PPE set is agreed and available for use by the PI team.

5.74. In the PI scenario, the extent to which PPE will remain in place may be equally as important as the infection control specification. This may result in the need for a riskassessed balance between equipment that may provide optimum infection control performance and equipment which is likely to remain in place, is comfortable and does not represent other hazards. When there is a choice between types of PPE, the visual impact of the PPE on the patient, who may be distressed, should be considered.

5.75. It is also recognised that different organisations may have different availability and specification of equipment. It is therefore important the best use is made of the equipment that is available.

\section{PPE shown as viable in physical intervention test scenarios}

5.76. The advice below is provided on the basis of a systematic evaluation of PPE performance during PI (Appendix 2). It should be considered in concert with, but not supersede, any local arrangements.

5.77. A range of available PPE was evaluated to offer insights into their performance characteristics in the PI context. This included their infection control contact contamination characteristics associated with PI.

5.78. The PPE below, used collectively, has shown acceptable performance in test episodes of managing aggressive behaviour using PI trainers (for details of tests undertaken see Appendix 2).

\section{Recommended physical intervention PPE set}

5.79. The following are recommended:

- Goggle-type eye protection with adjustable strap and face sealing capacity. There are googles available with a nylon elasticated strap which has shown to be effective in keeping the protection in place. This may provide less than optimal infection control properties and will therefore require specific procedures for cleaning or quarantine.

It is important that mitigation against misting is employed, either within the design of the eye protection or by using anti-misting spray. 
- Face mask. The highest specification for liquid resistance and filtration available to the unit is recommended meeting the standards of FFP3.

- Second layer of clothing. Disposable scrubs have shown acceptable performance and are recommended.

- Protective gloves. The highest specification protective gloves available to the unit are recommended.

- Disposable cleansing wipes. For post-PI cleansing, including head and face as described above (5.64-5.67).

5.80. All items should be subject to further infection control guidance for the donning and doffing, disposal and where appropriate the cleaning and storage of reusable equipment.

5.81. Wherever possible, reusable PPE should be allocated for use by a named member staff to reduce the potential for infection from equipment used by more than one staff member.

\section{SEARCHING}

6.1. Searching of mental health inpatients is governed by law and practice in this area should be consistent with Chapter 8 of the Mental Health Act Code of Practice (Department of Health 2015) ${ }^{12}$

6.2. Before any search takes place, consent should be sought.

6.3. Searching should be proportionate and the minimum intrusion necessary to maintain health, safety and a therapeutic environment.

6.4. Some search procedures require physical contact e.g. rub down procedures and property searches.

6.5. Searches should be undertaken using appropriate PPE which should include gloves and a fluid resistant mask.

6.6. When undertaking standard search procedures, unless absolutely necessary, contact should be avoided with the hands and face of the person being searched.

6.7. The person being searched should be asked to cleanse their hands prior to the search.

12 Department of Health (2015) Mental Health Act 1983: Code of practice.

https://www.gov.uk/government/publications/code-of-practice-mental-health-act-1983 
6.8. Wherever possible, close proximity between the heads of the person undertaking the search and the person being searched should be avoided.

\section{Stand-off searching devices}

6.9. For detection of some restricted items, hand wands can be used. These have the advantage of reducing the amount of physical contact required, although may produce false alerts or not detect some items.

6.10. Magnetic anomaly detection scanners have proved effective in detecting a range of restrictive items (Laidlaw et al. 2017) ${ }^{13}$ and do not require any contact between the person being searched and the operator. Searches can be undertaken at a distance which where possible may reduce the risk of infection.

\section{REFERENCES}

BGS (2020) Coronavirus: Managing delirium in confirmed and suspected cases. British Geriatrics Society. Updated 25 Mar. https://www.bgs.org.uk/resources/coronavirusmanaging-delirium-in-confirmed-and-suspected-cases

CQC (2020) Monitoring the Mental Health Act in 2019/20: The Mental Health Act in the COVID-19 pandemic. Care Quality Commission.

https://www.cqc.org.uk/sites/default/files/20201127_mhareport1920_report.pdf

Department of Health (2015) Mental Health Act 1983: Code of practice.

https://www.gov.uk/government/publications/code-of-practice-mental-health-act-1983

Laidlaw, J., Dix, R., Slack, P., Foy, C., Hayward, A., Metherall, A., Ireland, S., Wright, J., O'Rourke, P., Williams, J. and Rose, A. (2017) Searching for prohibited items in mentalhealth hospitals: a randomised controlled trial of two metal-detecting technologies. Medicine, Science \& the Law, 57(4): 167-174. https://doi.org/10.1177/0025802417725642

Morawska, L. and Milton, D.K. (2020) It is time to address airborne transmission of coronavirus disease 2019 (COVID-19). Clinical Infectious Diseases, 71(9): 2311-2313. https://doi.org/10.1093/cid/ciaa939

NHS England (2020a) Key actions: infection prevention and control and testing. 17 Nov. https://www.england.nhs.uk/coronavirus/publication/key-actions-infection-prevention-andcontrol-and-testing/

${ }^{13}$ Laidlaw, J., Dix, R., Slack, P. et al. (2017) Searching for prohibited items in mental-health hospitals: a randomised controlled trial of two metal-detecting technologies. Medicine, Science \& the Law, 57(4): 167-174. https://doi.org/10.1177/0025802417725642 
NHS England (2020b) Pulse oximetry to detect early deterioration of patients with COVID-19 in primary and community care settings. 11 Jun.

https://www.england.nhs.uk/coronavirus/wp-content/uploads/sites/52/2020/06/C0445remote-monitoring-in-primary-care-revised.pdf

NICE (2020a) COVID-19 rapid evidence summary: acute use of non-steroidal antiinflammatory drugs (NSAIDs) for people with or at risk of COVID-19. Evidence Summary ES23. 14 Apr. https://www.nice.org.uk/advice/es23/chapter/Factors-for-decision-making NICE (2020b) COVID-19 rapid evidence summary: vitamin D for COVID-19. Evidence Summary ES28. 29 Jun. https://www.nice.org.uk/advice/es28/chapter/Advisory-statementon-likely-place-in-therapy

Patel, M.X., Sethi, F. et al. (2018) Joint BAP NAPICU evidence-based consensus guidelines for the clinical management of acute disturbance: de-escalation and rapid tranquillisation. Journal of Psychiatric Intensive Care, 14: 89-132. https://doi.org/10.20299/jpi.2018.008 Zhu, Y., Chen, L., Ji, H., Xi, M., Fang, Y. and Li, Yi (2020) The risk and prevention of novel coronavirus pneumonia infections among inpatients in psychiatric hospitals. Neuroscience Bulletin, 36: 299-302. https://doi.org/10.1007/s12264-020-00476-9 


\section{Contributors/consultees}

Dr David Straiton, MRCPsych Psychiatry Speciality Doctor

Dr Peter Metherall PhD Clinical Scientist, Sheffield Teaching Hospitals

Jules Haste, Principal Pharmacist

Lisa McLean, RGN Senior Infection Control Nurse

Andy Hayward, HND PMVA Senior Tutor

Gary Ginger, RN Behaviour Support \& Training Team Lead

Louise Forrester, RMN, RGN, Lead Nurse for Infection Control for Mental Health \& Learning Disability

Paul O'Rourke, Senior Physical Intervention \& Reducing Restrictive Practice Instructor

Rob Jefferies, MSc Reducing Restrictive Practice Lead

Alan Metherall, Associate Director of Nursing

Caroline Parker, Consultant Pharmacist

Laura Woods, Consultant Nurse

Bernard Fox, Expert by Experience

Dr Jim Laidlaw, Consultant Psychiatrist

Dr Hamid Alhaj, Consultant Psychiatrist

Dr Aileen O’Brien, Consultant Psychiatrist

Dr Dinal Vekaria, Consultant Psychiatrist

Andy Johnston, Specialist Clinical Advisor, Mental Health

Cath Gamble, Mental Health Profession Lead, RCN

Geoff Brennan, Chief Executive BRIGHT, Executive Director, STAR WARDS

RCPsych Quality Network PICU

Matthew Page, Chief Operating Officer, AWP

Alison Bussey, Chief Nurse, MPFT

Peter Pratt, Specialist Pharmacist

Emma Wadey, Head of MH Nursing, NHSE/I

Garry Dodson, Matron

Steve Ireland, Matron

Laura Pensom, Ward Manager

Joy Duxbury, Professor of Mental Health

Guy Cross, Regulatory Policy Officer, CQC

Dr Keith Reid, Consultant Psychiatrist 
NAPICU Executive Committee

Jimmy Noak, Director of Nursing Broadmoor Hospital

Simon Linsey, Senior Specialist Solicitor

Dr Wendy Sherwood, Consultant Occupational Therapist

Jyoti Sharma, Infection Control Lead Nurse, AWP 


\section{NAPICU Administration Office}

Scottish Enterprise Technology Park

Nasmyth Building

2nd Floor

60 Nasmyth Avenue

East Kilbride

Glasgow

G75 0QR

Tel: 01355244585

info@napicu.org.uk

https://www.napicu.org.uk

Published by NAPICU International Press

(C) NAPICU 2020

doi: https://doi.org/10.20299/napicu.2020.001 


\section{Primary}

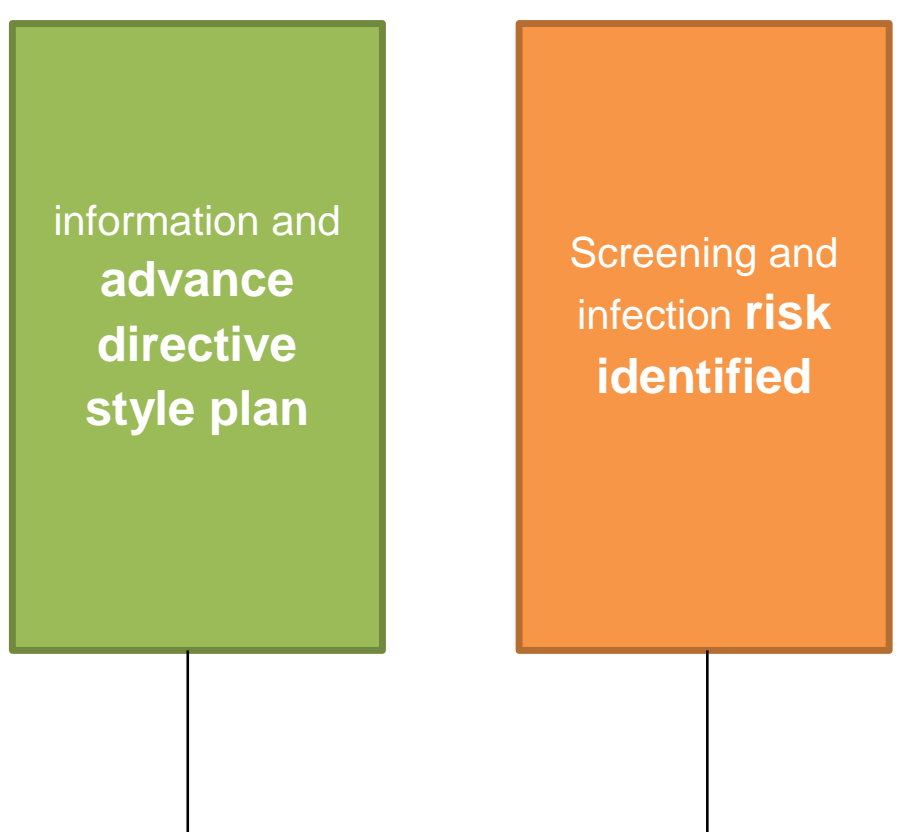

\section{Tertiary}

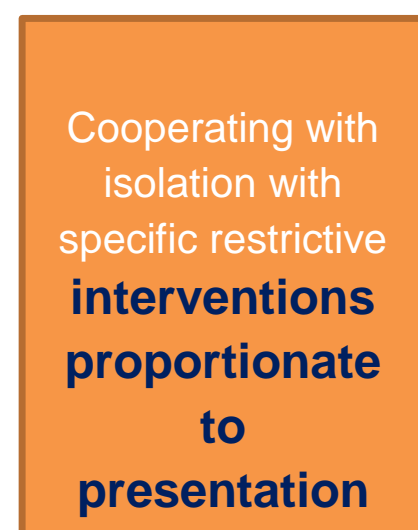

Significant

infection risk,

unable to

cooperate

although not

actively resisting

hierarchy of

response
Serious risk of infection, reckless

or deliberate

infection-related

behaviour requiring

segregation /

seclusion

monitoring 


\section{Appendix 2: PPE in the context of supporting a disturbed patient who is suspected or confirmed COVID-19 positive}

\section{Introduction}

The following represents preliminary data from a systematic evaluation of the performance of PPE during physical intervention (PI). The results are currently in review for publication (Dix et al. in review ${ }^{1}$ ).

Episodes of PI require very close physical contact between staff and patient. There is also potential for physically challenging struggle during which opportunities for contact transmission of COVID-19 are increased.

The following are common characteristics of PI which may be considered to significantly increase the risk of COVID-19 and other infections:

- Bodies are in physical contact with each other particularly hands providing direct opportunity for contact contamination

- The need for any PPE to remain in place and able to withstand high levels of demanding physical activity.

- The potential for any PPE to be purposely damaged or attempts at removal made by the recipient of PI

- Potential for very close proximity between the heads (particularly the oral and nasal region) of those involved in $\mathrm{Pl}$; this can range from $50 \mathrm{~mm}$ to $500 \mathrm{~mm}$

- Potential for very close proximity directed projection of larger droplets of oral fluid e.g. by spitting

- Potential for very close proximity shouting, coughing and raised voice projecting smaller particles of respiratory and oral secretions.

- Increased respiratory rate and depth resulting from physical exertion increasing the possibility of secretion and/or inhalation of virus-containing material.

\footnotetext{
1 Dix, R, Straiton, D., Metherall, P., Laidlaw, J., McLean, L., Hayward, A. et al. (in review) COVID-19: a systematic evaluation of personal protective equipment (PPE) performance during restraint.
} 


\section{Testing}

\section{Contact contamination}

Twelve participants, divided into three groups of four, simulated three episodes of PI. Each of the episodes involved one participant who represented the infection source (patient) and the remaining three represented members of a PI team (staff). In each episode the simulated PI followed a predetermined choreography and in each episode the group playing the role of staff were dressed in a different set of PPE (Fig. 1).

Figure 1. PPE sets evaluated.

\begin{tabular}{|l|l|l|}
\hline \multicolumn{1}{|c|}{ PPE Set A } & \multicolumn{1}{|c|}{ PPE Set B } & \multicolumn{1}{c|}{ PPE Set C } \\
\hline $\begin{array}{l}\text { - Protective goggles } \\
\text { - Coveralls } \\
\text { - FFP3 mask }\end{array}$ & $\begin{array}{l}\text { - Protective goggles } \\
\text { - Disposable scrubs } \\
\text { - Gloves }\end{array}$ & $\begin{array}{l}\text { - Fluid resistant surgical } \\
\text { mask } \\
\text { - Fluid resistant surgical } \\
\text { mask }\end{array}$ \\
\hline
\end{tabular}

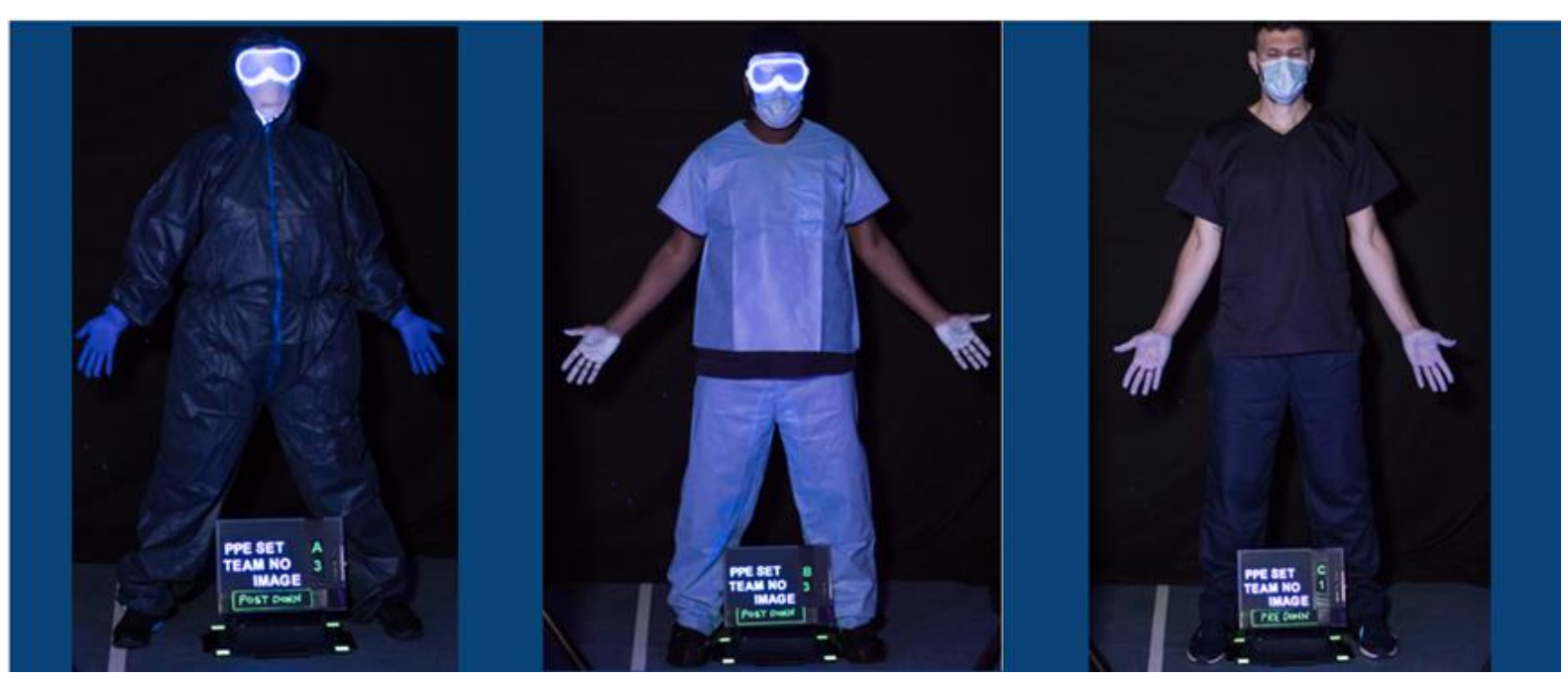

\section{Contamination analysis}

Comparison of the simulated contamination was made between the different PI episodes and contamination types using a standardised analysis of the UV photographs. Established deformable image registration techniques (MIM Software, Cleveland $\mathrm{OH}$ ) were implemented to align all visible light photographs for the definition of nine standard regions (head, neck, four thorax regions, arms, hands and legs). These were then applied to the UV photographs to measure the regional distribution of the contamination.

Detection of the coloured dyes was performed by segmenting the UV photographs using a clustering methodology ( $k$-means clustering with UV images converted to the CIE L*a* ${ }^{*}{ }^{*}$ 
colour space; Chebbout \& Merouani 2012²). Here the dominant colour of each dye (and other background features) was found from photographs of the infection source test subjects prior to the PI. These colours were then used to partition the test staff member photographs to identify areas of contamination. Combining this segmentation with the regional body contours permits a zonal analysis. Some manual editing was required to exclude regions of clothing which appeared blue in the UV image.

The participant representing a patient infected with COVID-19 had UV material placed in the areas of the body most likely to contain infectious material; i.e. lower face, upper chest, arms and hands. Spitting of oral fluid was simulated using ultraviolet (UV) fluorescent material consistent with training aids for infection control.

\section{Spitting}

At two points during the PI episodes spitting was simulated:

- Point One: spray bottle $150 \mathrm{~mm}$ away from the target

- Point Two: spray bottle $300 \mathrm{~mm}$ away from the target.

Following the PI episode, the amount and location of contact transfer of UV fluid was tracked using computerised image analysis.

\section{Non-contact contamination characteristics of PPE performance}

The following were also subject to evaluation:

- The extent to which the PPE remained in place

- Potential or actual hazards arising from the PPE

- The ease with which the different PPE sets could be donned and doffed

- Comfort of the PPE during use.

\section{Physical intervention simulation}

The following PI procedures were choreographed and enacted in three episodes as a linear progressive sequence of similar time duration ( 4.5 minutes):

- Immediate containment of assault

- Relocation

- De-escalation.

${ }^{2}$ Chebbout, S. and Merouani, F.H. (2012) Comparative study of clustering based colour image segmentation techniques. Eighth International Conference Signal Image Technology \& Internet Based Systems (SITIS). 2012; 839-844. https://doi.org/10.1109/SITIS.2012.126 


\section{Preliminary results}

Non-contact contamination characteristics of PPE performance

Table 1. Non-contact contamination characteristics of PPE performance

\begin{tabular}{|l|l|l|l|}
\hline PPE & Remain in place & Hazards & Comfort \\
\hline Goggles & $\begin{array}{l}\text { No clear issues observed } \\
\text { No explanation for spit on eye; } \\
\text { possible fit or frame failure }\end{array}$ & $\begin{array}{l}\text { Quickly misted up } \\
\text { severely restricting } \\
\text { vision }\end{array}$ & $\begin{array}{l}\text { Some discomfort } \\
\text { after } 20 \text { mins }\end{array}$ \\
\hline FFP3 & No issues & No issues & No issues \\
\hline FRM & No issues & No issues & No issues \\
\hline Coveralls & $\begin{array}{l}\text { Zip marginally opened during } \\
\text { the PI procedures }\end{array}$ & $\begin{array}{l}\text { Caused high levels } \\
\text { of heat and } \\
\text { perspiration }\end{array}$ & $\begin{array}{l}\text { Very uncomfortable } \\
\text { from overheating }\end{array}$ \\
\hline Disposable & $\begin{array}{l}\text { Can rip on donning if not } \\
\text { properly sized } \\
\text { scrubs }\end{array}$ & No issues & No issues \\
\hline Scrubs & $\begin{array}{l}\text { No issues } \\
\text { Tanped during PI }\end{array}$ & No issues & No issues \\
\hline
\end{tabular}

Table 2. Time taken for donning and doffing

\begin{tabular}{|l|l|l|}
\hline PPE Set & Donning & Doffing \\
\hline A & 5 minutes 32 seconds & 4 minutes 06 seconds \\
\hline B & 4 minutes 2 seconds & 4 minutes 19 seconds \\
\hline C & NA & 3 minutes 12 seconds \\
\hline
\end{tabular}




\section{Contact contamination}

All three of the PPE sets showed similar performance in the amount of transfer of contaminant. Pre-doffing, the location of containment was similar between the teams.

Figure 2. Post-physical intervention UV contamination, PPE sets $A, B$ and C.

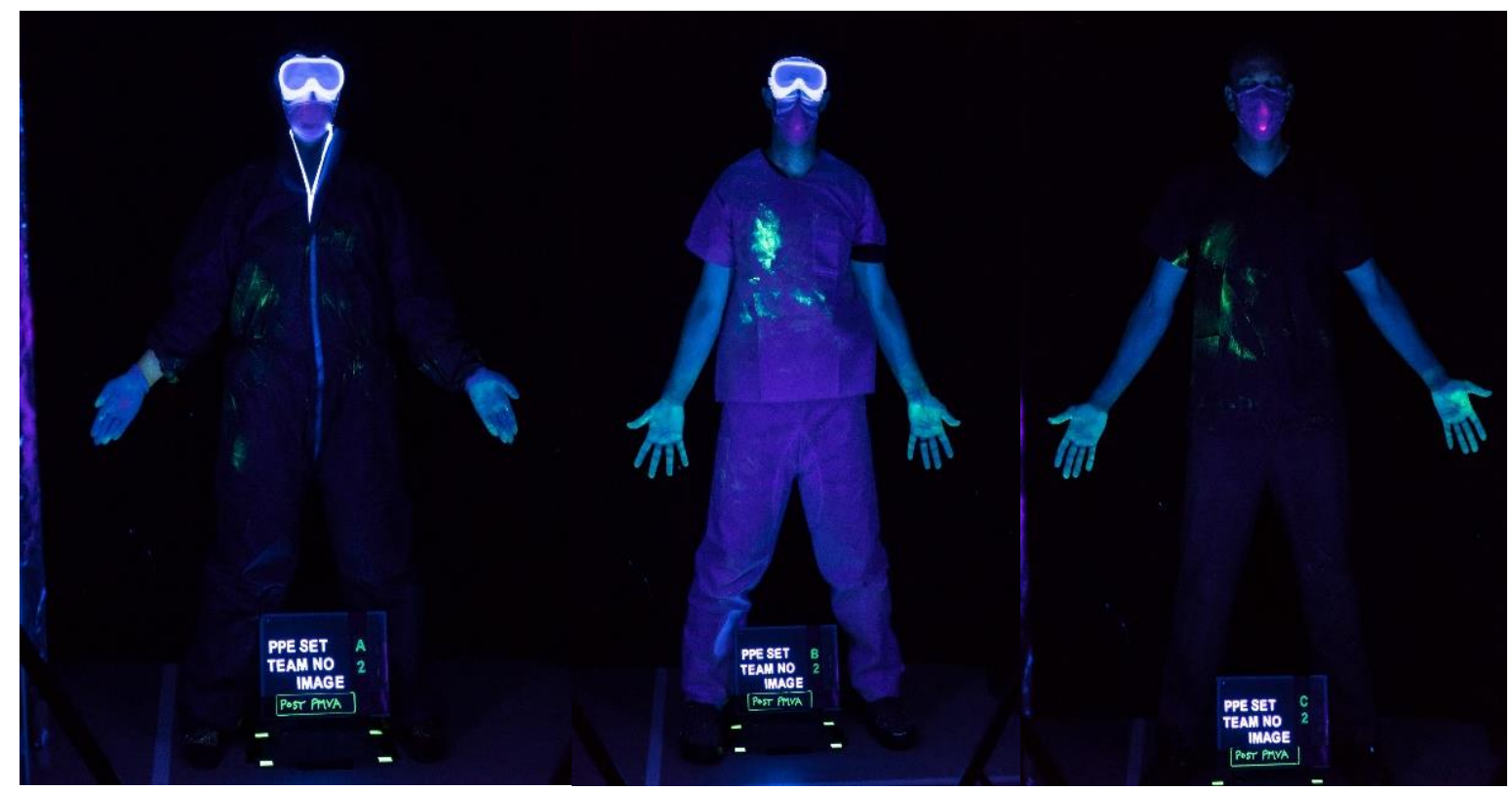

Only PPE Set A (disposable coveralls) provided protection of the arms. This set showed less UV contaminant in contact with skin in the arms area post PI compared to Set B and Set C.

Of particular note was contaminant located in axilla region (coloured green) of numbers 2 and 3 in the PI teams. This arose from contact with the infection source hands and arms when they were secured using PI techniques. Numbers 2 and 3 (left and right side of the patient) in the teams received less contamination (coloured blue), compared to team member 1 originating from the face and chest area of the infection source.

Figure 3. UV contamination on physical intervention team members 2 and 3.

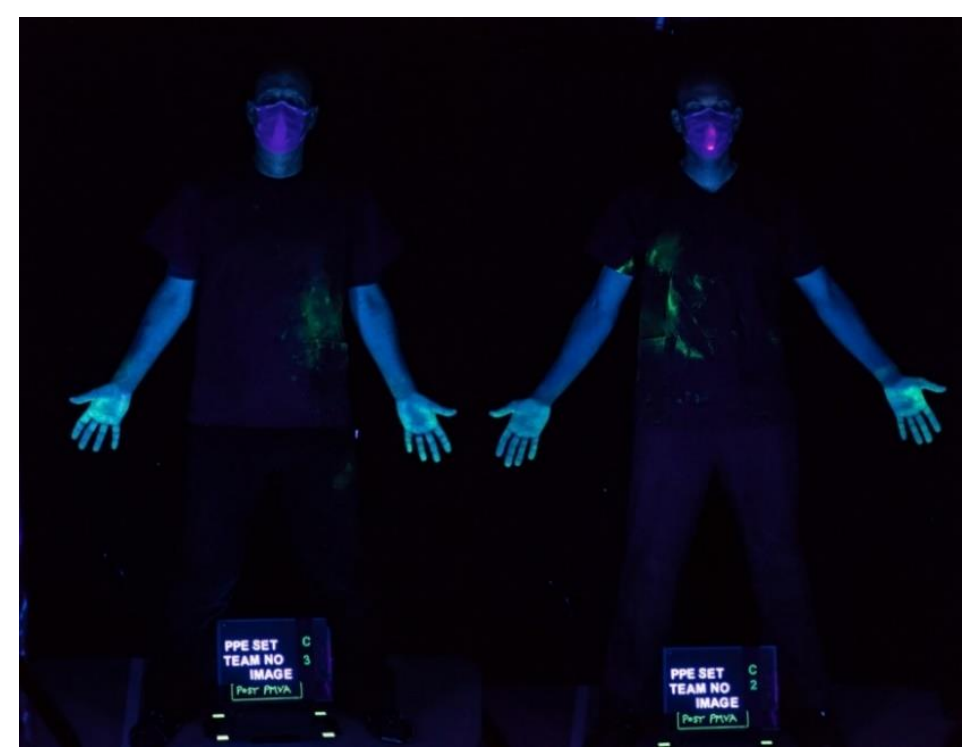


PI team member 1 (responsible for the controlling the head) had more UV material than the other team members on their hands and wrist, which had transferred from the face and torso area (coloured blue) of the infection source. Number 1 in each team received significantly less contamination from the hands and arms of the infection source (coloured green) than the other two team members.

Figure 4. UV contamination on hand and arm.
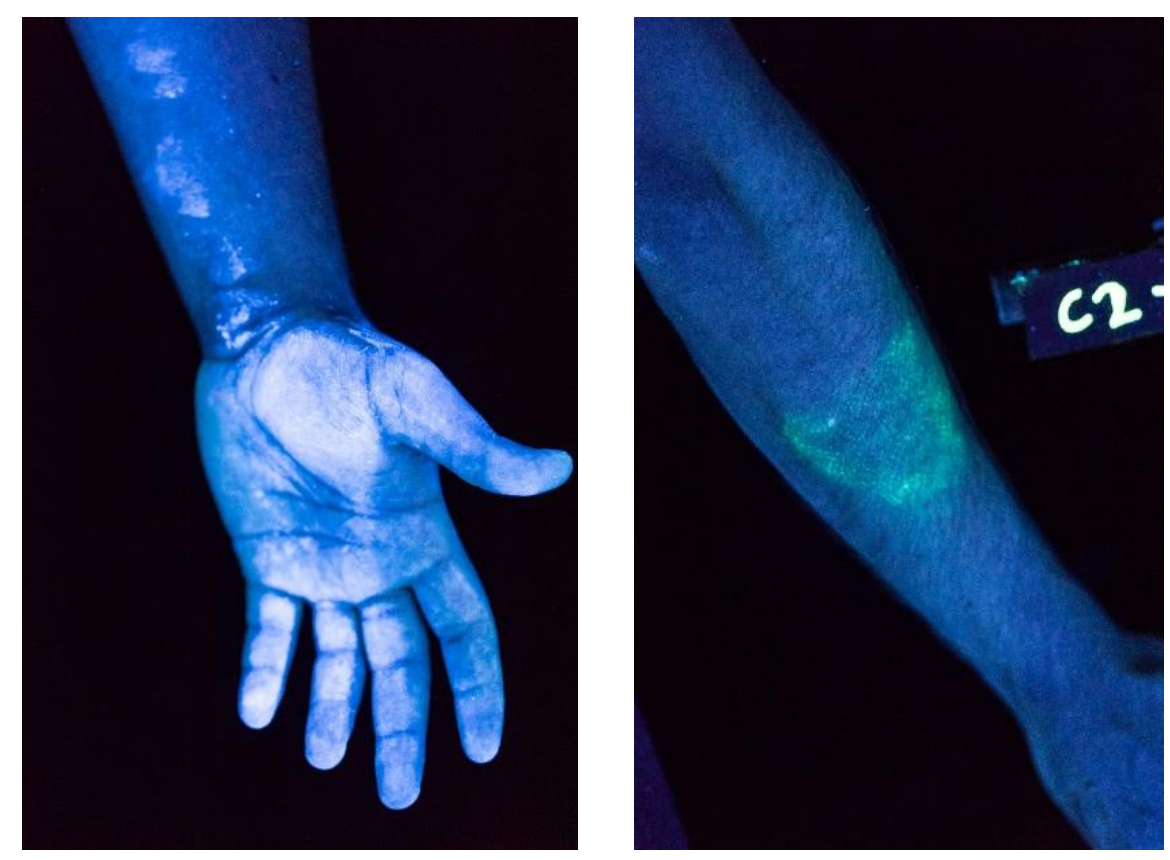

Following doffing, all three PPE sets showed similar performance in protecting against contamination transfer. For teams not using coveralls, this was significantly dependent upon effective cleansing as part of doffing. One of the test subjects clearly missed cleansing an area of contact contamination on the arms. If the PPE had provided arm coverage, it is likely that this area would not have remained contaminated post doffing. 


\section{Spitting}

The spitting simulation (coloured red) presented particular challenges for the PPE. Both the fluid resistant and the FFP3 mask were successful at preventing contact contamination. However, spit contaminant did breach the goggles and contact the lower eye area of the one of the test subjects. Contaminant was also found on the neck area and the automatic analysis identified small regions over the torso in a number of subjects.

Figure 5. UV material from simulated spit around eye and mask
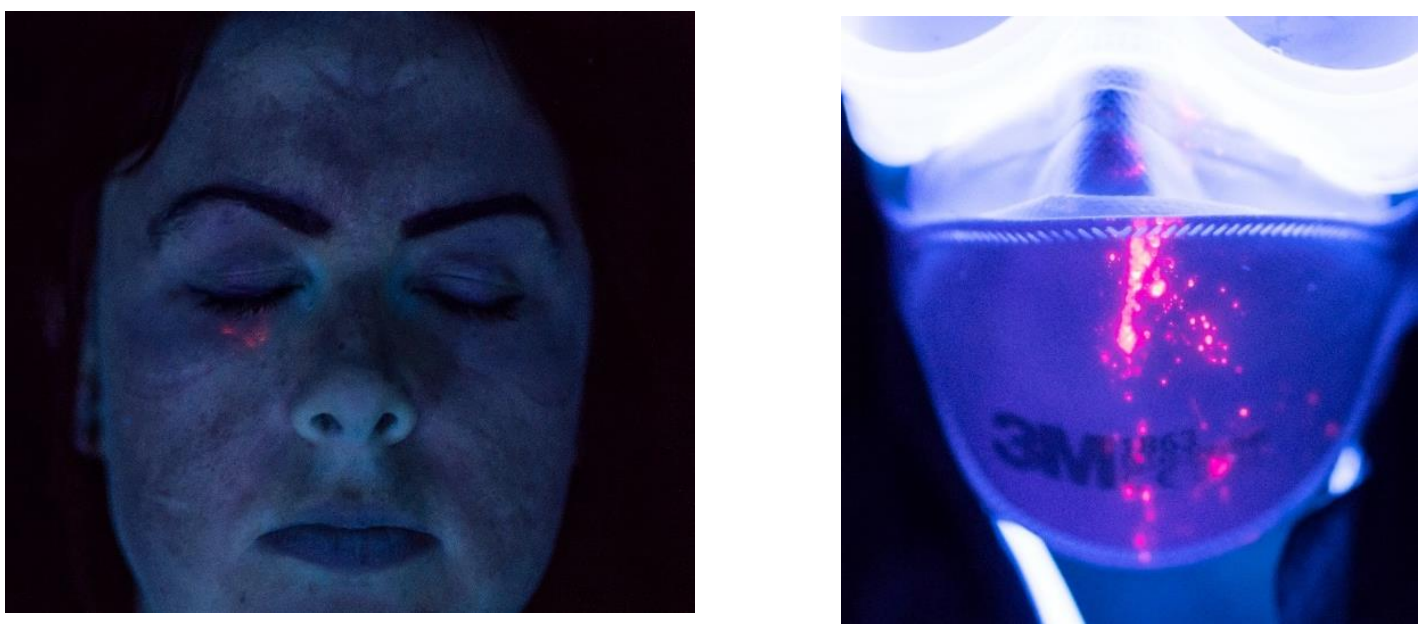

Careful review of the video recording could not clearly establish how the contaminate breached the goggles. Theories include the front panel of the goggles becoming dislodged from the frame or the goggles face seal becoming displaced during the PI episode.

Spitting demonstrated the need for face and neck cleansing procedures that currently do not feature in standard cleansing advise for health care.

\section{Non-contact contamination characteristics of PPE}

These experiments demonstrated that PPE needed to be properly fitted to the wearer.

Examples of this include the coverall zips travelling downwards during simulation as a result of being oversized for the individual. There was also an episode of disposable scrubs ripping on donning as a result of them being too small for the test subject's legs.

Two areas of major concern include the overheating experienced by the test subjects from the coveralls. The goggles misted up to the point of severely restricting the vision of the PI team members. 